\title{
Um roteiro de entrevista clínica centrada na pessoa para a graduação médica
}

\author{
A patient-centered clinical interview script for medical undergraduate teaching
}

\section{Un guion de entrevista clínica centrado en la persona para el pregrado en medicina}

Leandro David Wenceslau', Victor Kelles Tupy da Fonseca', Luíza de Alcântara Dutra', Letícia Gonçalves Caldeira'

${ }^{1}$ Universidade Federal de Viçosa (UFV), Viçosa, MG, Brasil.

\section{Resumo}

O ensino de habilidades de comunicação clínica na graduação médica encontra nos princípios e componentes do Método Clínico Centrado na Pessoa (MCCP) uma referência importante para a definição de suas competências. No entanto, mesmo tendo contato com o MCCP em sua formação, é frequente que estudantes de medicina recorram à utilização da anamnese tradicional centrada na agenda médica como um roteiro mais seguro para realização de suas entrevistas. Propomos, como uma hipótese para essa dificuldade dos estudantes, a falta de uma tradução do MCCP em um roteiro padronizado de entrevista médica, especialmente para ensino na graduação. Neste relato, a partir de modelos de entrevista clínica centrada na pessoa (ECCP) selecionados da literatura internacional, apresentamos a primeira etapa de um roteiro de ECCP original, adaptado ao cenário brasileiro. $O$ objetivo deste relato é oferecer uma referência de fácil utilização em língua portuguesa e que possa ser aprimorada pelos profissionais envolvidos com o ensino de comunicação clínica na educação superior no Brasil. Estudos empíricos ainda são necessários para endossar uma utilização mais ampla da proposta aqui apresentada.

Palavras-chave: Educação Médica, Anamnese, Cuidado Centrado no Paciente

\begin{abstract}
The teaching of clinical communication skills in medical undergraduate studies finds in the principles and components of the Patient-Centered Clinical Method (PCCM) an important reference to define its competences. However, even having contact with the PCCM in undergraduate teaching, medical students often resort to the traditional medical doctor-centered anamnesis as a safer script for conducting their interviews. We propose, as a hypothesis for this difficulty, the absence of a PCCM translation into a standardized medical interview script, especially for undergraduate teaching. In this report, based on patient-centered clinical interview $(\mathrm{PCCl})$ models selected from the international literature, we present the first step of an original PCCI script, adapted for the Brazilian scenario. This report aims to provide a user-friendly reference in Portuguese that can be improved by professionals involved with the teaching of clinical communication skills in higher education in Brazil. Empirical studies are still needed to support wider use of the proposal presented here.
\end{abstract}

Keywords: Medical Education, Medical History Taking, Patient-Centered Care

Como citar: Wenceslau LD, Fonseca VKT, Dutra LA, Caldeira LG. Um roteiro de entrevista clínica centrada na pessoa para a graduação médica. Rev Bras Med Fam Comunidade. 2020;15(42):2154 https://doi.org/10.5712/rbmfc14(41)2154
Autor correspondente: Leandro David Wenceslau. E-mail: leandro.david@ufv.br Fonte de financiamento: declaram não haver. Parecer CEP: não se aplica. Conflito de interesses: declaram não haver. Recebido em: 24/07/2019. Aprovado em: 25/10/2019. 


\section{Resumen}

La enseñanza de las habilidades de comunicación en el pregrado en medicina encuentra en los principios y componentes del Método Clínico Centrado en la Persona (MCCP) una referencia importante para la definición de sus competencias. Sin embargo, incluso teniendo contacto con el MCCP en su formación, es frecuente que los estudiantes de medicina recurran al uso de la anamnesis tradicional centrada en la agenda médica, como un guion más seguro para realizar sus entrevistas. Proponemos, como una hipótesis para esta dificultad de los alumnos, la falta de una traducción del MCCP en un guion estandarizado de entrevista médica, especialmente para la enseñanza en el pregrado. En este relato, a partir de modelos de entrevista clínica centrada en la persona (ECCP) seleccionados de la literatura internacional, presentamos el primer paso de un guion de ECCP original, adaptado al escenario brasileño. El objetivo de este relato es ofrecer una referencia en lengua portuguesa de fácil utilización y que puede ser mejorada por los profesionales involucrados en la enseñanza de la comunicación clínica en la educación superior en Brasil. Todavía se necesitan estudios empíricos para respaldar un uso más amplio de la propuesta que aquí se presenta.

Palabras clave: Educación Médica, Anamnesis, Atención Dirigida al Paciente

\section{Introdução}

As diretrizes curriculares dos cursos de medicina no Brasil ${ }^{1}$ estabelecem, desde 2014, a formação em atenção primária à saúde (APS) e nos cuidados centrados na pessoa como parte fundamental dos currículos nacionais de graduação médica. Esta orientação tem lançado o desafio, para as escolas médicas brasileiras, de reverem seus currículos e implementarem mudanças que incorporem a formação em competências para a APS, explorando suas interseções e contribuições para a formação nos demais níveis de atenção.

Já em 2013, no curso de medicina da Universidade Federal de Viçosa, foi proposta a implementação de uma disciplina voltada para o ensino de um conjunto de habilidades clínicas básicas para a realização de atendimentos médicos, em especial na Atenção Primária à Saúde. Essa iniciativa de ensino foi corroborada pelas diretrizes curriculares de 2014 e, desde então, seus conteúdos, métodos de ensino e avaliação têm sido desenvolvidos e aprimorados em um trabalho em equipe de docentes e preceptores.

Com exceção das competências semiológicas (abordadas em outras disciplinas do seu currículo), essas habilidades foram agrupadas em três grandes temas - comunicação clínica, raciocínio clínico e introdução à prática clínica baseada em evidências. Foram designadas, dado o seu caráter transversal na clínica contemporânea, não restrito à APS, de "metaferramentas da prática clínica". Neste relato de experiência, descrevemos a primeira parte do roteiro de entrevista clínica centrada na pessoa (ECCP) que vem sendo utilizado como eixo das técnicas de comunicação trabalhadas nesta disciplina.

Para definir essas competências, foi tomado como principal subsídio teórico o Método Clínico Centrado na Pessoa (MCCP), desenvolvido por Stewart et al. $^{2}$ e seus quatro componentes: explorar a saúde, a doença e a experiência de doença; entender a pessoa como um todo; elaborar um plano conjunto de manejo dos problemas; e fortalecer a relação entre a pessoa e médico.

Também foram utilizados os consensos internacionais curriculares para ensino de habilidades de comunicação clínica de Toronto, ${ }^{3}$ de Kalamazoo ${ }^{4}$ e para a graduação médica no Reino Unido. ${ }^{5}$ Como apontado por Hashim, ${ }^{6}$ a centralidade do paciente em um atendimento médico é alcançada por meio de três objetivos: levantar a perspectiva do(a) paciente sobre o seu adoecimento, compreender o contexto psicossocial do(a) paciente e construir objetivos terapêuticos compartilhados baseados nos valores do(a) paciente. 
No entanto, assim como McWhinney afirma que "o método clínico usado pelos médicos é sempre a expressão prática de uma teoria médica"2 (p. 17), propomos que modelos de entrevista são, por sua vez, a tradução operacional do método clínico a ser adotado por médicos e profissionais de saúde em geral. Nesse sentido, em nossa experiência ao ensinar o MCCP, esbarramos na dificuldade de alunos de graduação de traduzir esse método clínico em um modelo de entrevista - um roteiro estruturado de perguntas, afirmações e técnicas de comunicação verbal e não verbal, que pudesse ser aplicado no treinamento das suas primeiras entrevistas clínicas.

Embora os componentes do MCCP fossem valorizados como objetivos da prática médica, observamos, com frequência, estudantes recorrerem, em suas consultas de aprendizagem, ao uso da coloquialmente chamada "anamnese convencional completa" como roteiro "de segurança" de sua entrevista, obedecendo suas etapas traduzidas em uma sequência de perguntas centradas na agenda médica - identificação, queixa principal, história da doença atual, história patológica pregressa, história familiar, história fisiológica e história psicossocial, muitas vezes acrescidos de uma inesgotável revisão de órgãos e sistemas. Esse modelo, ainda bastante reproduzido na formação médica, é designado por Frain ${ }^{7}$ como "o modelo tradicional de uma história do paciente estruturada" (tradução nossa).

Por outro lado, são de amplo conhecimento também, na medicina de família e comunidade, as obras voltadas ao desenvolvimento de métodos de consulta centrados no paciente como os propostos por Moulton, ${ }^{8}$ Neighbour, ${ }^{9}$ Pendleton et al., ${ }^{10}$ Ramos, ${ }^{11}$ e Kurtz et al., ${ }^{12}$ entre outros. Ainda que esses métodos de consulta apresentem diversas técnicas e intervenções de comunicação clínica, eles também não oferecem um modelo conciso de entrevista clínica mais adequado para a graduação médica e a aprendizagem inicial de entrevistas.

A obra "A Entrevista Clínica", ${ }^{13}$ de Borrell Carrió, reúne também um amplo conjunto de recursos para aprimoramento da comunicação na consulta médica; porém, são, em sua maior parte, conhecimentos e habilidades avançadas, segundo nossa avaliação, não suprindo a lacuna de uma proposta básica para ensino na graduação.

\section{Métodos e Resultados}

O reconhecimento dessa lacuna nos fez buscar, na literatura científica, modelos de entrevista mais do que de consulta - centrados na pessoa e que pudessem ser uma alternativa prática ao modelo tradicional. Considerando esse recorte, identificamos, na literatura internacional, duas referências principais que constituíram a base do modelo de entrevista proposto neste relato: os livros Smith's Patient-Centered Interviewing: An Evidence-Based Method ${ }^{14}$ e The Medical Interview: The Three Function Approach. ${ }^{15}$

$\mathrm{Na}$ elaboração do roteiro aqui apresentado, o modelo de entrevista de Smith's Patient-Centered Interviewing ofereceu uma estrutura inicial detalhada para a primeira parte da entrevista com as respectivas intervenções de comunicação necessárias. Desenvolvido inicialmente por pesquisadores da Universidade do Estado de Michigan, liderados pelo internista Robert C. Smith, o método de Smith possui 5 passos e 21 subpassos. Esse método foi avaliado positivamente em pesquisas empíricas quanto ao seu potencial de ser ensinado de forma eficiente e de ser replicado. ${ }^{16,17}$ Também foram demonstrados, em ensaios clínicos randomizados, efeitos positivos em diversas medidas de saúde e na satisfação de pacientes com sintomas medicamente inexplicados. ${ }^{18}$ 
Em The Medical Interview: The Three Function Approach, Steven Cole, professor emérito de psiquiatria da Universidade de Stony Brook, e Julian Bird, psiquiatra e então professor da Universidade de Londres, apresentam um modelo de entrevista baseado em tarefas, ou como designam os autores, em três funções. Esse modelo foi inicialmente elaborado por Bird ainda nos meados dos anos 70.

Mais tarde, Lazare et al. ${ }^{19}$ propuseram uma versão aprimorada que fundamenta aquela utilizada na parceria entre Cole e Bird nas edições de seu livro. As três funções de Cole-Bird são: construir a relação médico-paciente; avaliar e compreender os problemas trazidos pelo(a) paciente; e colaborar para o manejo desses problemas. Para a execução de cada uma dessas funções, os autores indicaram técnicas específicas de comunicação clínica, que utilizamos também como referência do ensino-aprendizagem em nossa disciplina.

Em nossa avaliação, os modelos de Smith e de Cole-Bird são complementares, bem como as técnicas que são apresentadas. Por um lado, a síntese em três funções estabelece um norte claro quanto aos objetivos da entrevista médica; ela nos oferece marcadores iniciais simples de controle de qualidade do principal procedimento realizado por médicos e médicas em sua vida profissional - as entrevistas: "realizei essas três funções? Se sim, em que momento da entrevista e de que maneira?". As principais técnicas descritas para a realização de cada uma das três funções também se configuram métodos bem delimitados para colocar em prática tarefas muitas vezes complexas, como a construção de relacionamentos terapêuticos. Por outro lado, o modelo de Smith tem como principal qualidade a oferta de um roteiro bem delimitado, especialmente para a primeira parte da entrevista, isto é, até o início do exame físico ou a avaliação de exames complementares.

Ao longo dos últimos cinco anos, temos testado esses modelos no ensino da entrevista médica centrada no paciente para estudantes do terceiro período (segundo ano) da graduação médica e identificamos a necessidade de fazer modificações nas propostas originais. O roteiro aqui descrito apresenta diferenças que envolvem desde adaptações culturais até troca de ordem de algumas perguntas, acréscimos e recortes mais adequados para a realização das três funções. A seguir, vamos apresentar e analisar alguns aspectos da primeira etapa do roteiro: estabelecer contato, explorar e avaliar os problemas trazidos pelo(a) paciente (Quadro).

\section{Discussão}

A seguir, destacamos e analisamos alguns aspectos centrais do roteiro apresentado e que o diferenciam do modelo de entrevista "tradicional".

1. Abertura da entrevista e identificação: nesta proposta de entrevista, a identificação inicial da pessoa que procura atendimento deve ser um momento breve, que levanta os dados essenciais de nome e idade, com o objetivo de estabelecer um primeiro contato e confirmar a identidade do (a) paciente. Ela pode ser complementada por uma segunda identificação realizada em outro momento da entrevista ou mesmo outra consulta, em que outras questões de caráter social e demográfico podem ser levantadas de acordo com sua contribuição para entender os problemas trazidos pela pessoa. Evita-se, assim, um interrogatório inicial de perguntas fechadas cuja utilidade ou não dependerá desses problemas, que, na entrevista, só serão conhecidos mais adiante. Considera-se também uma potencial urgência do paciente em poder falar o quanto antes sobre o que lhe está afligindo, que é negligenciada quando o início da consulta se torna uma enquete demográfica rígida. 
Quadro. Roteiro de entrevista clínica centrada na pessoa para ensino na graduação médica.

Modelo de entrevista clínica centrada na pessoa para a graduação médica

Etapa 1 - Estabelecer contato, explorar e avaliar os problemas trazidos pelo(a) paciente

1) Acolhimento: chamar o(a) paciente pelo nome (com atenção especial ao registro no prontuário sobre uso de nome social em pacientes transgêneros); apresentar-se, dizendo sua função/papel no cenário clínico e nesta consulta; garantir uma acomodação adequada da pessoa no ambiente. Identificar e cumprimentar acompanhantes.

No caso de estudantes, perguntar pelo consentimento do paciente em ser atendido por estudante(s) e apresentar a forma como se dará a supervisão do atendimento.

2) Identificação: conferir apenas nome completo e idade do(a) paciente. Confirmar como o paciente deseja ser chamado(a) (novamente, estar atento à preferência pelo uso do nome social). Ratificar a concordância do paciente com a presença de acompanhantes durante a consulta.

3) 1' pergunta propiciatória: exemplos, "em que posso lhe ajudar hoje?", "o que lhe fez procurar esse serviço hoje?”, "me conta: o que lhe traz aqui hoje?". 4) Fala livre da pessoa: não interromper e utilizar técnicas não verbais de estímulo à fala do paciente (mão no queixo ou têmpora, mobilização afirmativa da cabeça, rosto e olhar e tronco voltados para o paciente, "peito aberto" e sem objetos entre vocês).

5) Checar demandas aditivas, até esgotá-las nesse primeiro momento: exemplos, "e que mais?" "algo mais Ihe preocupa quanto à sua saúde?" "você deseja uma avaliação sobre mais alguma questão hoje?" "há algo mais que você queira avaliar, solicitar ou trazer?" Perguntar até o paciente responder: "não."

6) Sumarização dos problemas trazidos pela pessoa: “Então, veja se eu entendi bem. Você nos procurou hoje por/para...."(usar as palavras do paciente para enumerar e descrever os problemas).

7) Pactuar agenda (definir o que vai ser abordado na consulta, de preferência, até dois problemas): priorizar de acordo com o que é mais importante para a pessoa, buscando conciliar com o que seja mais relevante do ponto de vista médico, incluindo informações de registros anteriores no prontuário que não tenham sido trazidas na fala livre.

Exemplo: "Em uma consulta médica de rotina, em geral, conseguimos avaliar de forma adequada um ou dois problemas. Certamente vamos programar a avaliação de todos trazidos por você. Mas qual seria a sua prioridade para essa nossa consulta de hoje? Reforçando que vamos agendar a avaliação dos demais."

"Dessas questões/situações que você me relatou, o que está te incomodando/preocupando mais?"

8) Explorar a experiência da doença (1): exploração narrativa livre com perguntas abertas iniciais (A):

"Conte-me mais sobre...(citar o problema pactuado na agenda, de preferência com as palavras do paciente)" "Como é esse/essa....?" "Como isso começou? Como tem sido/tem estado nos últimos dias?

Atenção: Observar o uso intencional do "como".

9) Explorar a experiência da doença (2): exploração dirigida dos componentes psicossociais do adoecimento:

Ideias (I): "o que você acha que pode ter causado isso?" "algo aconteceu com você, na sua vida, que você acha que tem a ver/pode ter causado esse problema?"

Preocupações (P): "tem algo nesse problema que está te preocupando mais?" "se está acontecendo desde ...., o que te fez buscar ajuda médica hoje?"

Funcionalidade (F): "como isso vem te atrapalhando no seu dia a dia?"

Expectativa (E): "e como você espera que eu possa te ajudar hoje quanto a esse problema?"

Sentimentos e afetos (S): "como você se sente em relação a esse problema?" "como ele tem te afetado?" "Como tem mexido com você?" OBS: Em geral, não são necessárias todas essas perguntas. Avalie o que já foi informado pelo paciente nas falas anteriores e faça, inicialmente, duas dessas perguntas que lhe pareçam mais úteis nesse momento.

Acrônimo da exploração da experiência de doença: AIPFES

10) Sumarização do que foi relatado pelo paciente até aqui sobre a experiência de adoecimento, confirmando um entendimento adequado do(s) problema(s).

11) Transição para agenda médica: "Agora vou lhe fazer mais algumas perguntas sobre esse problema para que eu possa entender um pouco melhor".

12) Histórico detalhado do problema pactuado: explorar os sete atributos do sintoma utilizando as informações já fornecidas pelo paciente. (1) Localização do sintoma, incluindo irradiação se for o caso, (2) cronologia ("quando começou? De lá pra cá, com que frequência, em que dias e horários, tem se manifestado?"), (3) tipo/qualidade, (4) intensidade, (5) fatores desencadeantes, de piora e de melhora, (6) sintomas associados, (7) tratamentos já experimentados.

13) Nova sumarização com síntese dos itens 9 e 12.

14) Histórico pregresso de problemas de saúde: Você está em tratamento para algum outro problema de saúde? Que outros problemas de saúde você teve/tratou? Faz ou já fez uso regular prolongado de algum medicamento (ser específico quanto a anticoncepcionais e medicamentos para hipertensão e diabetes)? Tem diabetes ou hipertensão? Já teve algum problema do coração ou derrame/"AVC"? Se mulher e de acordo com faixa etária específica, perguntar pela data da última menstruação e, oportunamente, levantar a regularidade de rastreamento de câncer de mama e câncer de colo uterino. Também em momento oportuno, perguntar sobre atualização de vacinas, transfusões, cirurgias e internações prévias.

15) Primeiro mapeamento familiar, social e ocupacional oportuno: Com quem mais você mora? Como estão as coisas em casa? Você tem ou já teve alguma ocupação regular de trabalho (considerar o trabalho doméstico) e/ou estuda? Como está caminhando o estudo e o trabalho? Você participa de alguma atividade social regularmente: religião, esporte, grupo social, comunitário ou cultural? Se participa, essa participação é importante para você? Se não participa, teria interesse em participar?

16) Rastreamento oportuno de problemas familiares: seus pais e irmãos têm algum problema de saúde, fazem algum tipo de tratamento? Como está a saúde dos seus pais? Há alguma doença importante na sua família? Seus pais ou irmãos apresentam ou apresentaram: HAS? DM? "Infarto do coração", derrame? Já realizaram "cateterismo" ou cirurgia do coração? Algum deles ou delas já teve câncer de intestino, mama ou próstata?

17) Rastreamento oportuno de comportamentos potencialmente prejudiciais à saúde: Você fuma? Você bebe/faz uso de bebidas alcóolicas? Você é ou foi sexualmente ativo/tem ou teve em algum momento relações sexuais? Usa ou usou sempre preservativo nas relações? Faz uso de algum outro método contraceptivo ou para evitar doenças sexualmente transmissíveis? Se não, por qual motivo? 
2. Lista de problemas e agenda da consulta: outro elemento significativo do presente roteiro é a inserção da pactuação da agenda como componente essencial dessa primeira etapa da entrevista. Com seus itens "queixa principal" e "história da doença atual" - naturalizados no singular -, o modelo tradicional cria a falsa expectativa de que as pessoas que procuram atendimento médico só terão uma "queixa" e um diagnóstico por consulta e que serão objetivas quanto ao seu sofrimento e preocupações. Essa expectativa, rapidamente frustrada nas primeiras entrevistas na atenção primária, não condiz com a realidade especialmente nesses cenários, ${ }^{20} \mathrm{em}$ que devem ser abordados todos os problemas de saúde da população sob seus cuidados independentemente de idade, órgão ou sistema afetado. Ao mesmo tempo, a atenção a diversos problemas requer a oferta de espaço de fala, oportuno e protegido, para que o (a) paciente possam relatá-los, explicitando sua agenda oculta, e o planejamento da abordagem da lista de problemas, em uma ou mais consultas. ${ }^{21,22}$ Stuart et al. ${ }^{23}$ analisaram 185 consultas de médicos de família britânicos e observaram uma média de 2,1 preocupações levantadas por consulta. Médicos que haviam sido treinados para explorar demandas adicionais da consulta o fizeram em $92,6 \%$ das vezes, enquanto não treinados, apenas $7 \%$.

3. A abordagem da experiência de adoecimento: a inserção, como rotina, de perguntas que explorem a experiência de adoecimento também é uma característica importante das entrevistas centradas na pessoa e que apresenta algumas particularidades nesta proposta. Recomendamos, baseados no modelo de Smith, que ela anteceda a agenda médica específica (perguntas voltadas principalmente para possíveis diagnósticos orgânicos ou biomédicos presentes no problema). Mas nada impede que, após a pactuação da agenda e da primeira pergunta aberta ("A"), o entrevistador ou a entrevistadora siga para exploração dos atributos do sintoma e, na sequência, explore alguns dos componentes de IPFES. Quanto aos componentes a serem explorados na experiência de adoecimento, propomos uma síntese dos já conhecidos IPE (ideias, preocupações e expectativas) ${ }^{14}$ e SIFE (sentimentos, ideias, funcionalidade e expectativas). ${ }^{2}$ Com o acrônimo AIPFES, reforçamos a importância de começar com uma pergunta exploratória totalmente aberta e de abordar diretamente sentimentos e afetos, porém, deixando-os para o final desta abordagem por se tratar de uma pergunta usualmente entendida pelos pacientes como mais íntima e pessoal. Destacamos, também, que não se faz necessário explorar todos os itens do acrônimo. Dois, talvez um, desses levantamentos já serão suficientes para que o (a) paciente possa trazer o que, de fato, está lhe causando sofrimento diante do problema priorizado.

4. Rastreamentos verbais oportunos: Um último aspecto a se destacar são os itens que classificamos como oportunos. No lugar das ortodoxas história familiar, história fisiológica, história psicossocial e revisão de órgãos e sistemas, propomos a utilização de componentes de rastreamento oportuno. São perguntas fechadas para levantamento de problemas e diagnósticos que, usualmente, não fazem parte da agenda da pessoa e, não necessariamente, precisam ser abordadas na primeira consulta, mas que, assim que possível e adequado, devem ser levantadas no contato longitudinal com o (a) paciente. A seleção dessas questões deve seguir recomendações de rastreamentos em população saudável de acordo com o perfil epidemiológico da pessoa. O modelo apresentado para este relato é voltado para adultos (18 a 60 anos) de ambos os sexos e baseado nas recomendações de Zonta et al. ${ }^{24}$ Esta costuma ser a população entrevistada, em geral, por alunos de graduação médica em suas primeiras entrevistas clínicas, embora os itens 1 a 12 do roteiro possam ser utilizados em consultas de qualquer faixa etária. 


\section{Conclusão}

Roteiros e modelos não devem limitar o exercício profissional, mas servir como uma base em constante aperfeiçoamento para facilitar o aprendizado e garantir a execução adequada de uma tarefa frequente e essencial de uma determinada profissão. O objetivo deste relato não é oferecer uma proposta hermética ou uma solução única para os complexos desafios do ensino da entrevista e da comunicação clínica, mas trazer uma referência de fácil utilização em língua portuguesa e com adaptações para o contexto brasileiro das graduações em medicina. Esperamos que sirva como base ou apoio para modelos melhores que diversos colegas e instituições possam desenvolver.

O treinamento de profissionais de saúde para tornar sua prática clínica mais centrada nas pessoas tem se demonstrado eficaz e sido correlacionado com desfechos positivos na satisfação, em comportamentos e no estado de saúde de diversas populações estudadas, embora a heterogeneidade dos estudos dificulte conclusões mais quantificáveis e categóricas. ${ }^{25}$ Nesse sentido, é nosso interesse também realizar estudos empíricos que avaliem a centralidade da pessoa do modelo apresentado neste relato e sua factibilidade de aprendizagem, que são necessários para endossar um uso mais extensivo na educação médica no Brasil.

\section{Referências}

1. Ministério da Educação (BR), Conselho Nacional de Educação, Câmara de Educação Superior. Resolução №. 3 de 20 de junho de 2014. Institui diretrizes curriculares nacionais do curso de graduação em Medicina e dá outras providências. Brasília: Diário Oficial da União; 2014. p. 8-11.

2. Stewart M, Brown JB, Weston WW, McWhinney IR, McWilliam CL, Freeman TR. Medicina Centrada na Pessoa:Transformando o Método Clínico. $3^{\text {a }}$ ed. Porto Alegre: Artmed; 2017.

3. Simpson M, Buckman R, Stewart M, Maguire P, Lipkin M, Novack D, et al. Doctor-patient communication: the Toronto consensus statement. BMJ. 1991;303(6814):1385-7.

4. Makoul G. Essential elements of communication in medical encounters: the Kalamazoo consensus statement. Acad Med. 2001;76(4):3903.

5. Noble LM, Scott-Smith W, O'Neill B, Salisbury H; UK Council of Clinical Communication in Undergraduate Medical Education. Consensus statement on an updated core communication curriculum for UK undergraduate medical education. Patient Educ Couns. 2018;101(9):1712-9.

6. Hashim MJ. Patient-Centered Communication: Basic Skills. Am Fam Physician. 2017;95(1):29-34.

7. Frain J. Why Clinical Communication Matters? In: Cooper N, Frain J, eds. ABC of Clinical Communication. Hoboken, NJ:Wiley Blackwell; 2018.

8. Moulton L. The Naked Consultation: A Practical Guide to Primary Care Consultation Skills. $2^{\text {nd }}$ ed. Boca Raton, FL: CRC Press; 2016.

9. Neighbour R. The Inner Consultation: How to Develop an Effective and Intuitive Consulting Style. Lancaster Lancashire, Boston: MTP Press; 1987.

10. Pendleton D, Schofield T, Tate P, Havelock P. A Nova Consulta - desenvolvendo a comunicação entre médico e paciente. Porto Alegre: Artmed; 2011.

11. Ramos V. A consulta em 7 passos: Execução e análise crítica de consultas em medicina geral e familiar. Lisboa: VFBM Comunicação; 2008.

12. Kurtz S, Silverman J, Benson J, Draper J. Marrying content and process in clinical method teaching: enhancing the Calgary-Cambridge guides. Acad Med. 2003;78(8):802-9.

13. Borrell Carrió F. Entrevista Clínica: Habilidades de Comunicação para Profissionais de Saúde. Porto Alegre: Artmed; 2012.

14. Fortin AH, Dwamena FC, Lepisto BL, Frankel RM, Smith RC. Smith's Patient-Centered Interviewing: An Evidence-Based Method. $4^{\text {th }}$ ed. New York: McGraw-Hill Education; 2019. 
15. Cole SA, Bird J. The Medical Interview: The Three Function Approach. $3^{\text {rd }}$ ed. Philadelphia, PA: Elsevier/Saunders; 2014.

16. Smith RC, Fortin AH, Dwamena F, Frankel RM. An evidence-based patient-centered method makes the biopsychosocial model scientific. Patient Educ Couns. 2013;91(3):265-70.

17. Smith RC, Lyles JS, Mettler J, Stoffelmayr BE, Van Egeren LF, Marshall AM, et al. The effectiveness of intensive training for residents in interviewing. A randomized, controlled study. Ann Intern Med. 1998;128(2):118-26.

18. Smith RC, Lyles JS, Gardiner JC, Sirbu C, Hodges A, Collins C, et al. Primary care clinicians treat patients with medically unexplained symptoms: a randomized controlled trial. J Gen Intern Med. 2006;21(7):671-7.

19. Lazare A, Putnam SM, Lipikin M Jr. Three function of the medical interview. In: Lipkin M Jr, Putnam SM, Lazare A, Carroll JG, Frankel RM, Keller A, et al., eds. The Medical Interview: Clinical Care, Education, and Research. New York: Springer-Verlag; 1995.

20. Hunziker S, Schläpfer M, Langewitz W, Kaufmann G, Nüesch R, Battegay E, et al. Open and hidden agendas of "asymptomatic" patients who request check-up exams. BMC Fam Pract. 2011;12:22.

21. Bjørland E, Brekke M. What do patients bring up in consultations? An observational study in general practice. Scand J Prim Health Care. 2015;33(3):206-11.

22. Silverman J. Hidden agendas and how to uncover them. Medicine. 2005;33(2):27-9.

23. Stuart B, Leydon G, Woods C, Gennery E, Elsey C, Summers R, et al. The elicitation and management of multiple health concerns in GP consultations. Patient Educ Couns. 2019;102(4):687-93.

24.Zonta R, Norman AH, Tesser CD, Galhardi MP, Capeletti NM. Rastreamento, Check-Up e Prevenção Quaternária. Florianópolis: UFSC; 2017.

25. Bradford N. Promoting a patient-centred approach in clinical consultations: Summary of a Cochrane review. Int J Nurs Pract. 2017;23(3). doi: $10.1111 /$ ijn. 12564 